Research Article

\title{
Deformation Control in Rest-to-Rest Motion of Mechanisms with Flexible Links
}

\author{
Roberto Caracciolo, Dario Richiedei, and Alberto Trevisani \\ Dipartimento di Tecnica e Gestione dei Sistemi Industriali (DTG), Università degli Studi di Padova, Padova, Italy \\ Correspondence should be addressed to Alberto Trevisani; alberto.trevisani@unipd.it
}

Received 31 August 2017; Revised 12 January 2018; Accepted 8 February 2018; Published 11 March 2018

Academic Editor: Kumar V. Singh

Copyright (C) 2018 Roberto Caracciolo et al. This is an open access article distributed under the Creative Commons Attribution License, which permits unrestricted use, distribution, and reproduction in any medium, provided the original work is properly cited.

\begin{abstract}
This paper develops and validates experimentally a feedback strategy for the reduction of the link deformations in rest-to-rest motion of mechanisms with flexible links, named Delayed Reference Control (DRC). The technique takes advantage of the inertial coupling between rigid-body motion and elastic motion to control the undesired link deformations by shifting in time the position reference through an action reference parameter. The action reference parameter is computed on the fly based on the sensed strains by solving analytically an optimization problem. An outer control loop is closed to compute the references for the position controllers of each actuator, which can be thought of as the inner control loop. The resulting multiloop architecture of the DRC is a relevant advantage over several traditional feedback controllers: DRC can be implemented by just adding an outer control loop to standard position controllers. A validation of the proposed control strategy is provided by applying the DRC to the real-time control of a four-bar linkage.
\end{abstract}

\section{Introduction}

The increasing effort towards improving dynamic performances and energy efficiency in machines and manufacturing systems is leading to the reduction of the moving masses. However, decreasing link masses also reduces their stiffness and causes elastic vibrations and deformations, which reduce system accuracy and precision [1] and cause large residual vibrations. Hence, motion planning and control of lightweight mechanisms and manipulators are two challenging issues. Traditional control schemes, such as standard PID (proportional-integral-derivative) regulators widely used in industrial controllers, have been demonstrated to be unsatisfactory to ensure effective compensation of unwanted elastic deformations. Therefore, attention should be paid to the synthesis of either optimal command generation techniques [2-4] or active vibration control schemes [5-11].

Optimal command generation techniques are feedforward approaches aimed at preplanning the control input on the basis of the system model, in such a way that the link elastic deformations are prevented. Their main advantage is the ease of implementation, since they just rely on the system model and no additional sensors are required. On the other hand, open-loop approaches usually have poor disturbance rejection and high sensitivity to model uncertainty and unknown initial conditions. Designing closedloop active control systems is therefore an effective way to cope with disturbances and model uncertainty. Several different techniques have been presented over the years. Most of these approaches take advantage of additional actuators, such as smart actuators embedded into flexible links $[5,6]$, or impose the modification of the torque, speed, or position control loops of the servo axes driving the systems [79]. However, these modifications are hard to implement in industrial controllers that are usually based on proprietary and closed architectures that cannot be modified. Among the few control techniques that do not impose modification of servo axis controllers, wave-based control $[10,11]$ and Delayed Reference Control [12] (DRC) should be mentioned. They both rely on the feedback-based modification of the position reference.

The DRC idea has been first proposed in [12] for vibrating linear systems with a single resonant mode and then extended in [13] to multi-DOF (Degree of Freedom) linear systems. 
The basic idea of DRC is to shape the desired position reference on the fly based on the measured values of the variables to be controlled, namely, the elastic deformations or oscillations. Vibration control is obtained by delaying or anticipating the original preplanned position reference if it causes elastic deformation. The preplanned desired input reference is not therefore defined explicitly as a function of time but rather as a function of a scalar time-varying "action reference parameter," which is in practice an environmentsensitive parameter computed on the basis of the measured deformations. DRC schemes can be adopted in those applications, where the major goal is performing a prescribed displacement accurately, with negligible elastic deformations, rather than a trajectory in a given time, since the motion time can be modified in a variable way. For the same reason, DRC schemes are sometimes referred to as "nontime-based" controllers, since the position reference is not explicitly defined as a function of the time. The concept of non-time-based control for dynamic systems, also referred to as "event-based" control, has been introduced in the 90s, and several successful controllers have been proposed thereafter, for instance, in neurosurgery [14], manufacturing processes [15], coordinated motion control [16], and robot control [17].

A peculiar feature of DRC schemes is that the trajectory planner assumes a primary role in the feedback closed loop, since it shapes the position references on the fly. Consequently, a multiloop control scheme is obtained, consisting of an inner position control loop and an outer loop. The former can be any standard position controller and is fed with the position reference computed by the outer loop. The latter includes the trajectory planner and is introduced to compute the action reference parameter for generating the suitable delayed position reference accomplishing the control goal. This architecture allows for the straightforward implementation of DRC schemes by simply adding an outer loop to standard position controllers, such as standard servo regulators employed in industrial automatic machines and manipulators. This is a relevant strength of the proposed method compared to most of the control techniques proposed in literature for deformation and vibration control, which impose the modification of the inner loop to perform vibration control.

In the numerical study [18], the DRC theory has been applied to the control of flexible link mechanisms in repetitive motion through numerical simulations. Starting from such a preliminary work, this paper proposes the first extension of the DRC to deformation control in rest-to-rest motion of flexible link mechanisms. The aim of the controller is to reduce the elastic deformations of the links, and therefore the method is suitable for those applications where this is a major requirement. The method is here developed with reference to an arbitrary flexible link mechanism in the presence of small deformations and then experimentally validated through a four-bar planar linkage with all the links being flexible. In particular, the crank and the follower deformations are controlled simultaneously by computing a scalar value of the action reference parameter, optimally trading between different control specifications.
Starting from a linearized model briefly described in Section 2, which provides a simplified but adequately accurate representation of the system dynamics, the method for synthesizing DRC schemes for curvature control is explained in Section 3. The experimental results validating the proposed approach, as well as the description of the test-bed and of the method implementation, are discussed in Section 4. Finally, concluding remarks are stated in Section 5.

\section{System Modelling}

Although the dynamics of flexible link mechanisms and manipulators is nonlinear and needs large dimensional models, the design of motion and vibration control schemes often relies on simplified, reduced-order, and linearized models. Indeed, it is widely recognized that, in the case of small deformations, the accuracy of linearized models about operating points is usually satisfactory enough to make their use successful in the synthesis of effective and stable control schemes. The neglected nonlinear and high-frequency dynamics can be treated, for example, as model uncertainty, which can be effectively tackled by paying attention to the controller robustness. All these considerations justify the use of a linearized model in the synthesis of the DRC scheme, which also makes the controller implementation easier by drastically reducing its computational effort and allowing for hard real-time control.

The model adopted is obtained by linearizing a nonlinear model accounting for the mutual coupling between large rigid-body motion and small elastic displacements. The model is valid for an arbitrary flexible link mechanism with holonomic and scleronomous constraints in the presence of small elastic displacements. The reader should refer to [9] and to the references therein for a more detailed description of such a model.

Independent coordinates have been adopted to represent the rigid-body motion, and the model is directly formulated through ordinary differential equations (ODE). As a matter of fact, this formulation allows for a more straightforward use of the control theory, which usually assumes this kind of model formulation.

The total motion of each flexible link is separated into the large motion of an equivalent rigid-link system (ERLS) behaving as a rigid moving reference mechanism whose number of DOFs is denoted as $r$ and the small elastic deflections of the links with respect to the ERLS named $\mathbf{u}$ whose size is denoted by $e$. Among the possible definitions of the rigid moving reference, the ERLS is defined in this work by imposing the fact that its generalized coordinates q coincide with the joint variables, which are also those measured and actuated to drive the mechanism. In this way, the actuators set the motion of the ERLS directly. The sum of $\mathbf{u}$ and the vector of the rigid positions of the node of the ERLS, denoted as $\mathbf{r}$, describes the motion of each node of the finite element model. By linearizing the nonlinear equations of motion obtained through the application of the Principle of Virtual Work, the following linear model is obtained by linearizing the nonlinear model about an equilibrium configuration set by the equilibrium values of the 
rigid displacement vectors and of the external force vector $\mathbf{v}$ $\left(\right.$ resp, $\left.\mathbf{q}_{\mathbf{e}}, \mathbf{v}_{\mathbf{e}}\right)$ :

$$
\begin{aligned}
& {\left[\begin{array}{cc}
\mathscr{M}_{\mathbf{e}} & \mathscr{M}_{\mathbf{e}} \mathbf{S} \\
\mathbf{S}^{T} \mathscr{M}_{\mathbf{e}} & \mathbf{S}^{T} \mathscr{M}_{\mathbf{e}} \mathbf{S}
\end{array}\right]_{\mathbf{q}=\mathbf{q}_{\mathbf{e}}}\left\{\begin{array}{l}
\ddot{\mathbf{u}}(t) \\
\ddot{\mathbf{q}}(t)
\end{array}\right\}} \\
& +\left[\begin{array}{cc}
\eta \mathscr{M}_{\mathbf{e}}+\lambda \mathscr{K}_{\mathbf{e}} & \mathbf{0} \\
\eta \mathbf{S}^{T} \mathscr{M}_{\mathbf{e}} & \mathbf{0}
\end{array}\right]_{\mathbf{q}=\mathbf{q}_{\mathbf{e}}}\left\{\begin{array}{l}
\dot{\mathbf{u}}(t) \\
\dot{\mathbf{q}}(t)
\end{array}\right\} \\
& +\left[\begin{array}{c}
\mathscr{K}_{\mathbf{e}} \\
\mathbf{0}-\left(\frac{\mathrm{d}\left(\mathbf{S}^{T} \mathscr{M}_{\mathbf{e}}\right)}{\mathrm{d} \mathbf{q}} \otimes \mathbf{g}+\frac{\mathrm{d} \mathbf{S}^{T}}{\mathrm{~d} \mathbf{q}} \otimes \mathbf{v}_{\mathbf{e}}\right)
\end{array}\right]_{\mathbf{q}=\mathbf{q}_{\mathbf{e}}} \\
& +\left\{\begin{array}{l}
\mathbf{u}(t) \\
\mathbf{q}(t)
\end{array}\right\}=\left[\begin{array}{c}
\mathbf{I} \\
\mathbf{S}^{T}
\end{array}\right]_{\mathbf{q}=\mathbf{q}_{\mathbf{e}}}\{\mathbf{v}\} .
\end{aligned}
$$

In (1), the following definitions have been adopted:

(i) $\mathscr{M}_{\mathrm{e}}$ and $\mathscr{K}_{\mathrm{e}}$ are the matrices obtained by assembling the mass and stiffness matrices of the finite elements.

(ii) $\mathbf{S}$ is the ERLS sensitivity coefficient matrix for all the nodes, relating the velocities of the ERLS generalized coordinates, $\dot{\mathbf{q}}$, to the velocities of all the nodes of the ERLS, $\dot{\mathbf{r}}: \dot{\mathbf{r}}=\mathbf{S}(\mathbf{q}) \dot{\mathbf{q}}$.

(iii) $\mathbf{I}$ and $\mathbf{0}$ are the identity and the null matrices.

(iv) The scalars $\eta$ and $\lambda$ are the Rayleigh damping coefficients.

$(\mathrm{v})(\mathrm{d} \mathbf{N} / \mathrm{dq}) \otimes \mathbf{b}$ denotes the inner product between arbitrary matrix $\mathbf{N}$ and vector $\mathbf{b}$, for all the subscripts $i, j,\left[\partial N_{i, 1} / \partial q_{j} \cdots \partial N_{i, n} / \partial q_{j}\right]$.

Admittedly, the linearized model holds only in a finite neighborhood of the working point; however, when large displacements are tackled, piecewise-linear models can be successfully employed to approximate the nonlinear system's dynamics better, as it has been proven in [9].

\section{Synthesis of the Delayed Reference Regulator}

3.1. Controller Specifications. The equations of motion in (1) are partitioned and expressed in the following more convenient form:

$$
\begin{gathered}
{\left[\begin{array}{ll}
\mathbf{M}_{\mathrm{EE}} & \mathbf{M}_{\mathrm{ER}} \\
\mathrm{M}_{\mathrm{RE}} & \mathrm{M}_{\mathrm{RR}}
\end{array}\right]\left\{\begin{array}{l}
\ddot{\mathbf{u}} \\
\ddot{\mathbf{q}}
\end{array}\right\}+\left[\begin{array}{ll}
\mathrm{C}_{\mathrm{EE}} & 0 \\
\mathrm{C}_{\mathrm{RE}} & 0
\end{array}\right]\left\{\begin{array}{l}
\dot{\mathbf{u}} \\
\dot{\mathbf{q}}
\end{array}\right\}} \\
+\left[\begin{array}{cc}
\mathrm{K}_{\mathrm{EE}} & \mathbf{0} \\
\mathbf{0} & \mathrm{K}_{\mathrm{RR}}
\end{array}\right]\left\{\begin{array}{l}
\mathbf{u} \\
\mathbf{q}
\end{array}\right\}=\left[\begin{array}{c}
\mathrm{I} \\
\mathbf{S}^{T}
\end{array}\right] \mathbf{v} .
\end{gathered}
$$

The subsystem of (2) comprising the first $e$ equations can be rewritten as the equations of motion of an $e$-DOF elastic system excited by the external nodal forces, collected in vector $\mathbf{v}$, and by the inertial coupling with the rigid-body motion, $\mathbf{M}_{\mathrm{ER}} \ddot{\mathbf{q}}(t)$ :

$$
\mathbf{M}_{\mathrm{EE}} \ddot{\mathbf{u}}(t)+\mathbf{C}_{\mathbf{E E}} \dot{\mathbf{u}}(t)+\mathbf{K}_{\mathrm{EE}} \mathbf{u}(t)=\mathbf{v}(t)-\mathbf{M}_{\mathbf{E R}} \ddot{\mathbf{q}}(t) .
$$

The right-hand side term of (3) can be therefore thought of as an equivalent force vector, henceforth referred to as $\mathbf{f}(t)$ :

$$
\mathbf{f}(t):=\mathbf{v}(t)-\mathbf{M}_{\mathbf{E R}} \ddot{\mathbf{q}}(t) .
$$

If the most critical vibrational modes are controllable, then $\mathbf{f}(t)$ can excite and control the link elastic displacements. In order to control the deformation, it is here suggested to make $\mathbf{f}(t)$ behave as a damping force. Hence, $\mathbf{f}(t)$ is required to be a function of the first derivatives of the measured strains of the link, $\dot{\mathscr{C}}(t)(\mathscr{C}(t)$ denotes the vector of the measured strains):

$$
\mathbf{f}(t)=-\int_{-\infty}^{+\infty} \mathbf{g}(t-\gamma) \dot{\mathscr{C}}(\gamma) d \gamma .
$$

In (5), $\mathbf{g}(t)$ represents both the DRC gains and the filters adopted to remove undesired signal components. Besides low-pass filters, adopted to remove noise, high-pass filters are employed to remove static components of strains.

The DRC idea is to make $\mathbf{f}(t)$ assume the desired values, defined in (5), by shaping $\ddot{\mathbf{q}}(t)$ (i.e., the time history of the rigid-body motion) rather than $\mathbf{v}(t)$. Hence, no active deformation control is explicitly introduced in the force vector $\mathbf{v}(t)$. This is similar to the approach followed by optimal planning techniques, where rigid-body motion is properly shaped to reduce oscillations. Compared with such methods, however, DRC is a feedback scheme and the optimal motion law is computed on the fly based on sensor measurements. The control problem is therefore stated as shaping the time history of the rigid-body motion $\mathbf{q}(t)$ in order to exert the desired active damping force defined through (5):

$$
\mathbf{v}(t)-\mathbf{M}_{\mathrm{ER}} \ddot{\mathbf{q}}(t)=-\int_{-\infty}^{+\infty} \mathbf{g}(t-\gamma) \dot{\mathscr{C}}(\gamma) d \gamma .
$$

Besides exerting an equivalent damping force, it is also required that the controller ensure correct tracking of the desired displacement (which sets the path in the case of systems with more rigid DOFs), defined regardless of the time of execution, rather than a trajectory in time as it happens in classical control schemes. To satisfy this second specification, the position reference of the rigid-body control loop $\mathbf{q}_{\text {ref }}$ is not made an explicit function of just the time but of a real scalar time-varying parameter $l(t)$ named "action reference parameter" [12]. The position reference will be henceforth referred to as $\mathbf{q}_{\text {ref }}(l)$. The action reference parameter is computed on the basis of the measured strains to exert the desired damping force $\mathbf{f}(t)$. The following definition of $l$ is chosen:

$$
l(t)=t-\tau(t)
$$

The real and scalar $\tau(t)$ is named "time delay," although it can assume also negative values (in other words, it can anticipate the execution of trajectory whenever necessary).

The $l$-domain value of the position reference $\mathbf{q}_{\text {ref }}(l)$ should be known explicitly, since it describes the ideal desired path to be tracked in the case of rigid bodies. In contrast, the time history of the position reference, referred to as $\mathbf{q}_{\text {ref }}^{*}(t)$, is not known a priori, since it depends on the time history of $l$, 
which in turn is computed on the fly through the measured strains.

The control problem is therefore stated as finding $\tau(t)$ such that the desired equivalent damping force in (5) is exerted and the actuator motion (which can be seen as the rigid-body motion) does not drift away from the desired displacement:

$$
\begin{array}{ll}
\tau(t) & \\
\text { s.t. } & \mathbf{v}(t)-\mathbf{M}_{\mathrm{ER}} \ddot{\mathbf{q}}(t)=-\int_{-\infty}^{+\infty} \mathbf{g}(t-\gamma) \dot{\mathscr{C}}(\gamma) d \gamma \\
& \mathbf{q}_{\text {ref }}^{*}(t)=\mathbf{q}_{\text {ref }}(t-\tau(t)) .
\end{array}
$$

3.2. Problem Solution. The control problem in (8) has no exact solution in the general case. However, some reasonable problem simplifications lead to an analytical solution. First of all, by assuming that the mechanism position controller ensures precise dynamic responses, in (6), the actual ERLS acceleration $\ddot{\mathbf{q}}(t)$ can be approximated by its reference value in time $\ddot{\mathbf{q}}_{\mathrm{ref}}^{*}(t)$. This assumption is common in most of the literature on optimal motion planning and therefore should not be considered restrictive. Secondly, the position reference may be linearized over a finite interval:

$$
\mathbf{q}_{\mathrm{ref}}^{*}(t)=\mathbf{q}_{\mathrm{ref}}(t-\tau) \simeq \mathbf{q}_{\mathrm{ref}}(t)-\boldsymbol{\beta} \tau(t),
$$

where the rigid-body reference velocity vector $\beta$ is assumed to be finite and constant over such an interval. Because of this assumption on $\boldsymbol{\beta}$, step inputs as position references cannot be employed if a DRC is used. Again, this assumption is not a severe drawback of the DRC, since step position references are not of interest in practice: indeed, only continuous position references, such as polynomials, are adopted in industrial applications. Hence, by replacing (9) and its first and second derivatives in (8), it holds that

$$
\begin{array}{r}
\mathbf{v}(t)-\mathbf{M}_{\mathrm{ER}} \ddot{\mathbf{q}}_{\mathrm{ref}}(t)+\mathbf{M}_{\mathrm{ER}} \beta \ddot{\tau}(t) \\
=-\int_{-\infty}^{+\infty} \mathbf{g}(t-\gamma) \dot{\mathscr{C}}(\gamma) d \gamma .
\end{array}
$$

The boundary conditions in (10) are set to ensure that if $\dot{\mathscr{C}}(t) \equiv 0$, then $\mathbf{f}(t) \equiv 0$ and $\tau(t) \equiv 0$ (i.e., $\ddot{\tau}(t)=0, \dot{\tau}(t)=$ $0 \forall t)$; this implies that if no undesired oscillations arise, no control force is exerted and therefore no delay is applied. Hence, if $\dot{\mathscr{C}}(t) \equiv 0$, then $\mathbf{v}(t)-\mathbf{M}_{\mathbf{E R}} \ddot{\mathbf{q}}_{\text {ref }}(t)=\mathbf{0}$. These boundary conditions lead to an overdetermined system made by $e$ independent differential equations in the scalar unknown $\ddot{\tau}(t)$ :

$$
\mathbf{M}_{\mathrm{ER}} \boldsymbol{\beta} \ddot{\tau}(t)=-\int_{-\infty}^{+\infty} \mathbf{g}(t-\gamma) \dot{\mathscr{C}}(\gamma) d \gamma .
$$

Equation (11) can be written in the frequency domain through the Laplace transforms of $\tau(t), \mathbf{g}(t)$, and $\mathscr{C}(t)$ (denoted as $T(s), \mathbf{G}(s)$, and $\mathbf{C}(s)$, resp.):

$$
\mathbf{M}_{\mathrm{ER}} \beta s^{2} T(s)=-s \mathbf{G}(s) \mathbf{C}(s) .
$$

A unique, scalar time delay satisfying simultaneously all these equations does not exist in general. The controller synthesis is therefore formulated as a least-square problem by looking for the scalar $\tau$ minimizing the norm of the residual in (12):

$$
\left\|\mathbf{M}_{\mathbf{E R}} \boldsymbol{\beta} s^{2} T(s)+s \mathbf{G}(s) \mathbf{C}(s)\right\|_{2} .
$$

Such a problem can be modified by weighing each equation of the system in (13) through a scalar-product matrix $\Omega$ to reflect different levels of concern about each control requirement. Additionally, an extra term $\left\|s^{2} T(s)\right\|_{2}^{2}$ is included in the minimization problem to avoid large and fast varying delays by penalizing its magnitude in the cost function:

$$
\left\|\boldsymbol{\Omega}\left\{\mathbf{M}_{\mathbf{E R}} \boldsymbol{\beta} s^{2} T(s)+s \mathbf{G}(s) \mathbf{C}(s)\right\}\right\|_{2}^{2}+\psi\left\|s^{2} T(s)\right\|_{2}^{2} .
$$

The scalar $\psi \geq 0$ trades off between the cost of missing the control specification and the cost of using large control effort. Since $\boldsymbol{\Omega}$ is defined as a positive-definite matrix, $\left\{\mathbf{M}_{\mathrm{ER}} \boldsymbol{\beta}\right\}^{T} \boldsymbol{\Omega}\left\{\mathbf{M}_{\mathrm{ER}} \boldsymbol{\beta}\right\} \geq 0 \forall \boldsymbol{\beta}$. Hence, the scalar $\left(\left\{\mathbf{M}_{\mathbf{E R}} \boldsymbol{\beta}\right\}^{T} \boldsymbol{\Omega}\left\{\mathbf{M}_{\mathbf{E R}} \boldsymbol{\beta}\right\}+\psi\right)^{-1}$ exists for any $\mathbf{M}_{\mathbf{E R}}$ and $\boldsymbol{\beta}$ and for any $\psi>0$. The solution is therefore

$$
T(s)=\frac{-1}{s} \frac{\left\{\mathbf{M}_{\mathrm{ER}} \boldsymbol{\beta}\right\}^{T} \boldsymbol{\Omega} \mathbf{G}(s)}{\left\{\mathbf{M}_{\mathrm{ER}} \boldsymbol{\beta}\right\}^{T} \boldsymbol{\Omega}\left\{\mathbf{M}_{\mathrm{ER}} \boldsymbol{\beta}\right\}+\psi} \mathbf{C}(s) .
$$

The extra term included in (14) is often denoted as "regularization term" [19]. The regularization problem significantly improves the numerical conditioning of the problem solution and increases its robustness with respect to the model variability and uncertainty [19].

Finally, the poles and zeros in $\mathbf{G}(s)$ due to the highpass filters adopted to get rid of the steady-state values of the strains are separated and made explicit through $\mathbf{G}_{0}(s)$ to obtain a more convenient form. In order to keep the controller implementation as simple as possible, it is here suggested to employ a first-order high-pass filter, that is, $\mathbf{G}(s):=\left(s /\left(s+I_{\text {cut }}\right)\right) \mathbf{G}_{0}(s)$, whose pole lies in $s=-I_{\text {cut }}$. The final formulation of the optimal time delay is therefore

$$
T(s)=\frac{-1}{s+I_{\text {cut }}} \frac{\left\{\mathbf{M}_{\mathbf{E R}} \boldsymbol{\beta}\right\}^{T} \mathbf{\Omega G}_{\mathbf{0}}(s)}{\left\{\mathbf{M}_{\mathbf{E R}} \boldsymbol{\beta}\right\}^{T} \boldsymbol{\Omega}\left\{\mathbf{M}_{\mathbf{E R}} \boldsymbol{\beta}\right\}+\psi} \mathbf{C}(s) .
$$

3.3. The DRC Multiloop Architecture. The DRC architecture is schematically described by the block diagram in Figure 1. The DRC scheme is based on a multiloop architecture made by an inner position loop (gray lines in Figure 1) and an outer loop (black lines) which run simultaneously. The "inner loop position controller" that can have any arbitrary architecture (such as those usually adopted in industrial controllers, as shown in Section 4.1) regulates the actuator to make $\mathbf{q}(t)$ track $\mathbf{q}_{\text {ref }}^{*}(t)$ as computed at each time step by the "position planner." The latter belongs to the outer loop, which implements the DRC equations. Hence, the outer loop is aimed at performing active control of the link deformation by computing suitable values of $l$ (which is calculated in the so-called action reference block) and therefore $\mathbf{q}_{\text {ref }}{ }^{*}(t)$. 
TABLE 1: Inertial and geometrical parameters of the four-bar linkage.

\begin{tabular}{|c|c|c|c|c|}
\hline Link & Frame & Crank & Coupler & Follower \\
\hline Length, $\mathrm{m}$ & 0.365 & 0.390 & 0.535 & 0.640 \\
\hline Flexural stiffness, $\mathrm{Nm}^{2}$ & \multicolumn{4}{|c|}{20.16} \\
\hline Cross-sectional area, $\mathrm{m}^{2}$ & \multicolumn{4}{|c|}{$36 e-6$} \\
\hline Joint & $A$ & $B$ & $C$ & $D$ \\
\hline Mass, kg & 0 & $14 e-2$ & $14 e-2$ & - \\
\hline Inertia, $\mathrm{kgm}^{2}$ & $2 e-4$ & 0 & 0 & $1.5 e-5$ \\
\hline
\end{tabular}

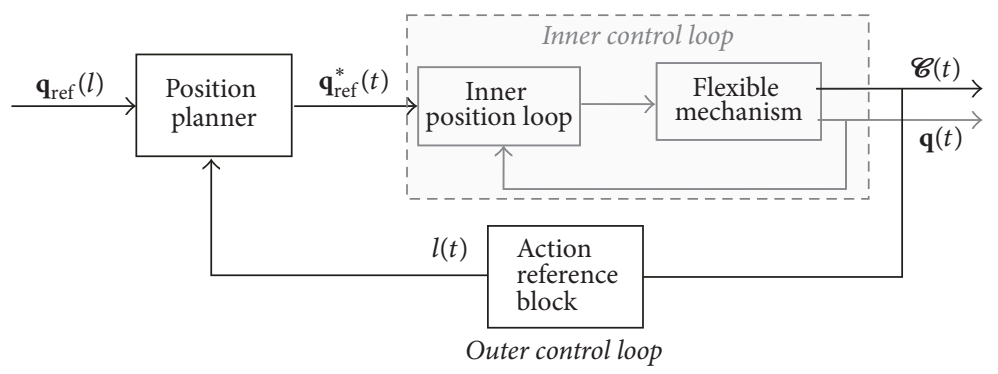

FIGURE 1: DRC multiloop architecture.

The real-time computation of $l$ at each time step is undemanding, since it just involves a first-order, singleinput-single-output linear filtering and some simple algebraic operations. In addition, the model linearization allows for the offline computation of matrices $\mathbf{M}$ and $\mathbf{S}$. All these operations can be efficiently performed by real-time controllers without incurring considerable computational costs.

\section{Experimental Application}

4.1. Implementation. A four-bar planar linkage with flexible steel bars has been developed (see Figure 2 and Table 1). The model adopted has 15 elastic DOFs $(e=15)$, and consequently $r+e=16$. The mechanism is actuated by an Indramat brushless servomotor directly driving the rotation of the crank $\alpha_{1}$ (which coincides with the ERLS generalized coordinate $q$ ) to track the delayed reference $\alpha_{1}^{\text {ref }}(l)=\alpha_{1}^{\text {ref }}(t-\tau)$, computed by the outer loop. The crank rotation is measured through an optical encoder with 20000 radial lines.

The motion control of the crank is performed by a standard position-velocity-current multiloop controller, as it is represented in Figure 3. The current (torque) and the speed control loops are performed by standard PI (proportionalintegral) regulators implemented in the Indramat controller (in Figure $3, K_{p v}$ and $T_{i v}$ denote the proportional gain and the integral action time of the speed controller, resp.). Velocity feedforward is also adopted to reduce the transient error by means of a filtered derivative of the position reference. A standard proportional controller, whose gain is $K_{p p}$, is also implemented in the position control loop to compute the speed reference for the motor drive on the basis of the crank angle tracking error. The speed and current loops are implemented in the proprietary Indramat controller, while the position control loop and the DRC outer loop

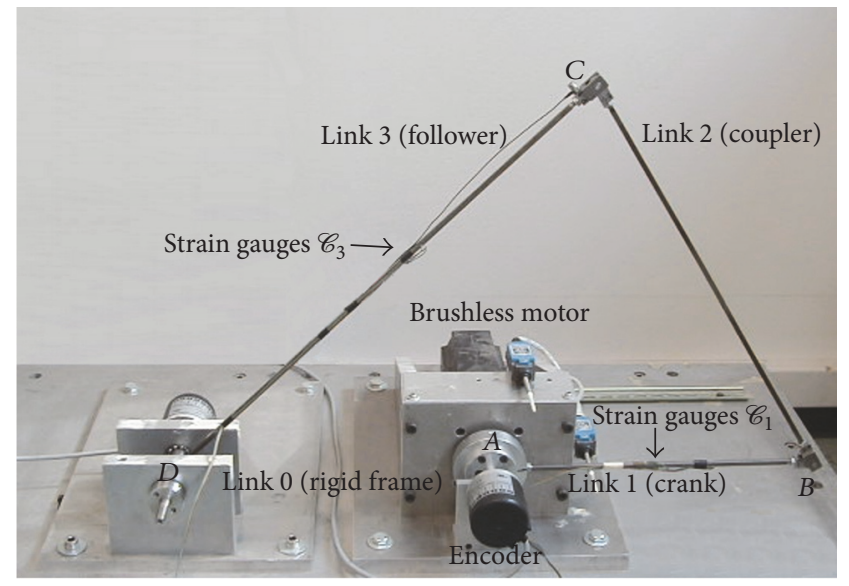

Figure 2: Picture of the mechanism.

are implemented in a Matlab-Simulink ${ }^{\circledR}$ code and run in an external target PC with xPC Target hard real-time operative system. The outer loop sample rate is $1 \mathrm{kHz}$ and a fourthorder explicit Runge-Kutta integrator is employed to solve (16). The ability of DRC schemes to deal with any arbitrary inner control loop is a strength of the DRC formulation and allows for DRC in industrial devices.

The DRC specification is to reduce the deformation of both the crank and the follower. Resistive strain gauges are therefore adopted (link $1, \mathscr{C}_{1}$, and link $\left.3, \mathscr{C}_{3}\right)$ to measure strains in the plane of motion.

An effective and simple choice for $\mathbf{f}(t)$ consists in an equivalent control torque computed through two constant gains $k_{\mathrm{C} 1}^{v}, k_{\mathrm{C} 3}^{v}$, that is, $\mathbf{G}(s)=\left[\begin{array}{lll}k_{\mathrm{C} 1}^{v} & k_{\mathrm{C} 3}^{v} \\ & 0\end{array}\right]\left(s /\left(s+I_{\text {cut }}\right)\right)$. Such gains have been tuned to equally weigh the requirements on the two links. In order to clarify the DRC impact on the 


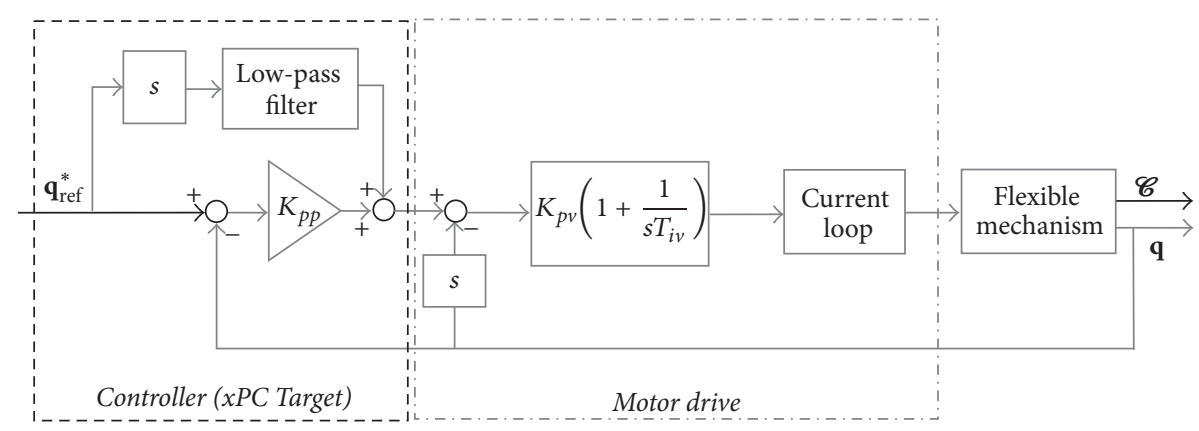

Figure 3: Inner control loop.

system dynamics, three different sets of DRC gains are tested (denoted as (a), (b), and (c)). The same inner loop is instead employed in all the tests. The results are also compared with the ones obtained through the sole inner loop. All the controllers share the same values of $I_{\text {cut }}=0.15 \mathrm{rad} / \mathrm{s}, \boldsymbol{\Omega}=\mathbf{I}$ (to equally weigh the requirements on the two links), and $\psi=1 e-4$, while they have different gains $k_{C 1}^{v}=k_{C 3}^{v}$ (resp., 160 for the controller "a," 125 for the controller "b," and 100 for the controller "c"). Higher gains result in more effective suppression of the link oscillations at the cost of larger delays.

The linearized model matrices in (16) have been computed by setting $q_{e}=0.35 \mathrm{rad}$ and are assumed to be constant over the entire range of motion to reduce the computational effort and memory allocation in the external target PC. These matrices provide accurate representation of the system dynamics in the range of interest for the test case proposed [18]. No switching between linear models has been adopted, but it could be done effectively in case it is necessary at the cost of an increase of the memory allocation and computational effort [20].

Finally, Table 2 lists the eighteen eigenvalues of the dynamics matrix of the augmented state closed-loop system, computed at the equilibrium through the method proposed in [18] and in the presence of the DRC controller "b." Similar results are obtained for the two other DRC controllers. The numerical pole analysis clearly proves that stability is assured with the proposed DRC, as confirmed by the experimental results shown in Section 4.2.

4.2. Experimental Results. The motion reference is a motion law with piecewise constant acceleration (trapezoidal speed profile), aimed at moving the crank from the horizontal configuration $\alpha_{1}^{\text {ref }}=0$ to the final one $\alpha_{1}^{\text {ref }}=0.7 \mathrm{rad}$ (an ideal motion time of $0.6 \mathrm{~s}$ has been assumed for the displacement with no delay in the case of rigid behaviour). Such a motion law is widely employed in industries for motion planning of rigid systems and is the most severe among the profiles used in practice. Therefore, it is not suitable for flexible mechanisms, since acceleration discontinuities (i.e., infinite jerk) cause relevant link deformations. Nonetheless, effective smoothing of such a trajectory in time is expected when DRC schemes are adopted. Indeed, the aim of the DRC is to shape the time history of the motion reference through $\tau$ to minimize the unwanted elastic deformation on the basis of the sensed strains.
TABLE 2: Closed-loop system eigenvalues.

\begin{tabular}{l}
\hline Eigenvalues \\
\hline$(1)-8.92$ \\
$(2)-38.17$ \\
$(3)-9123.7$ \\
$(4)-2.75 \pm 70.436 i$ \\
$(5)-2.89 \pm 324.10 i$ \\
$(6)-21.74 \pm 956.52 i$ \\
$(7)-35.50 \pm 229.33 i$ \\
$(8)-56.16 \pm 1347.6 i$ \\
$(9)-45.91 \pm 1438.7 i$ \\
$(10)-131.3 \pm 2309.3 i$ \\
$(11)-287.9 \pm 3554.2 i$ \\
$(12)-391.2 \pm 4133.2 i$ \\
$(13)-948.3 \pm 6400.8 i$ \\
$(14)-1824.4 \pm 8787.7 i$ \\
$(15)-2953.8 \pm 11027 i$ \\
$(16)-6612.7 \pm 15751 i$ \\
$(17)-23441.4 \pm 22030 i$ \\
$(18)-40601.7 \pm 12000 i$
\end{tabular}

Figure 4 shows the time history of the reference (black lines) and actual (grey lines) values of the crank angle obtained through the four controllers. These results highlight how the DRC outer loop shapes the crank reference by smoothing it through $\tau$ to minimize the elastic deformations. The higher the gains $k_{C 1}^{v}$ and $k_{C 3}^{v}$ (i.e., the more it is required to damp the link deformations), the larger the delay in the execution of the total displacement, as it is evident in the result provided by controller "a," which is the one with the highest gains. Smaller delays are obtained if smaller gains are employed. In particular, controller " $c$ " ensures the smallest rise time among the three DRC controllers evaluated. Figure 4 also reveals that all the controllers are asymptotically stable and hence confirms the theoretical expectations stated in Section 4.1 and in Table 2, based on the analysis of the closedloop system eigenvalues.

Figure 5 shows the time histories of $\tau$ and $l$. The peak values of the delay are $0.19 \mathrm{~s}, 0.42 \mathrm{~s}$, and $1.1 \mathrm{~s}$ for, respectively, controllers "a," "b," and "c." However, the modifications caused by $\tau$ have positive effects on the motion of the crank, 


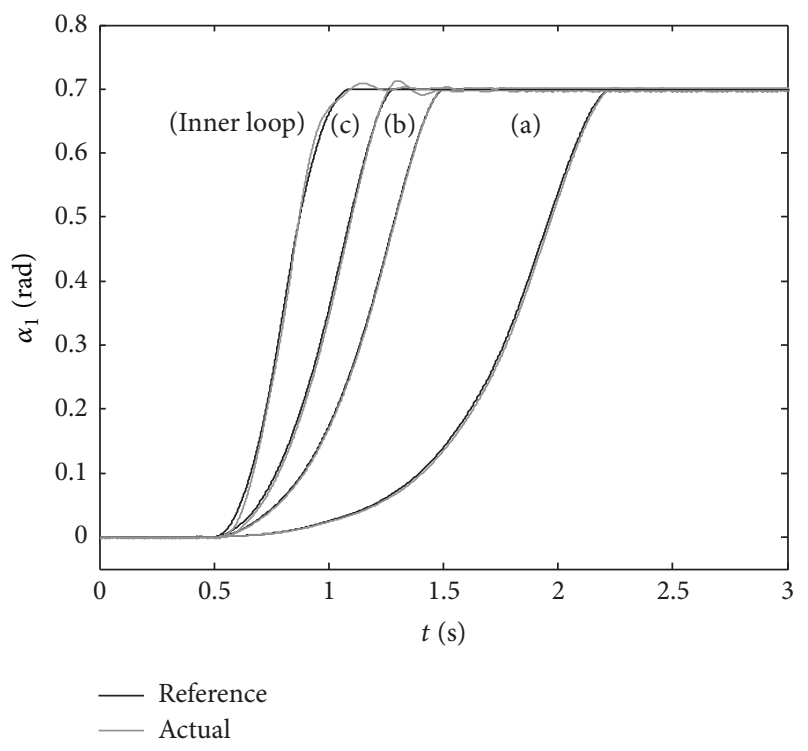

FIGURE 4: Reference and actual values of the crank angle versus time.
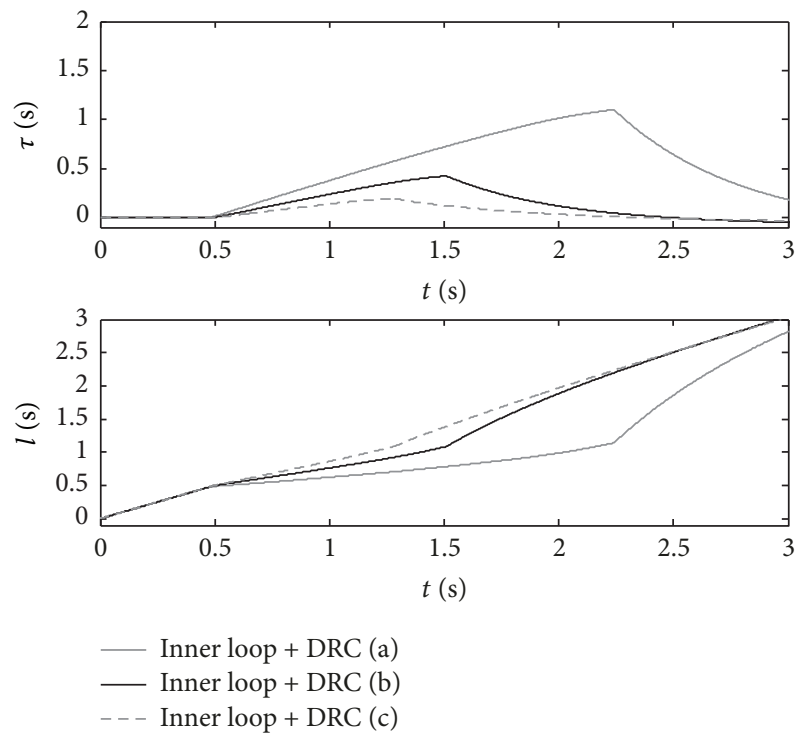

FIGURE 5: Time delay and action reference parameter versus time.

as revealed by the analysis of the rise time (i.e., the time required for moving from 10 to 90 percent of the final value of the crank displacement). Indeed, rise times of controllers "a" and "b" are $0.452 \mathrm{~s}$ and $0.565 \mathrm{~s}$, respectively, while the rise time if no DRC is employed is $0.298 \mathrm{~s}$. That means that the actual delays of the crank are smaller than those represented through $\tau$, and they are just $0.154 \mathrm{~s}$ and $0.268 \mathrm{~s}$, respectively. As a matter of fact, the elastic motion perturbs the "rigidbody motion" of the crank because of the dynamic mutual coupling already discussed in the model synthesis (see (1) and (2)). The deformation control exerted by the DRC controllers reduces these disturbances, thus allowing for better tracking of the references for the crank "rigid-body motion."

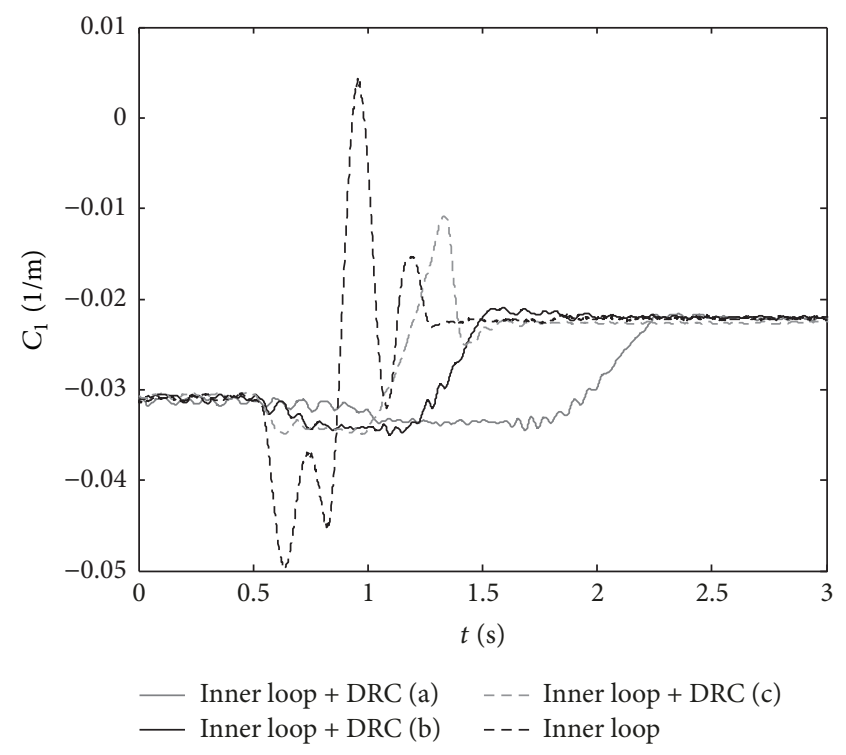

FIGURE 6: Comparison of the crank strains.

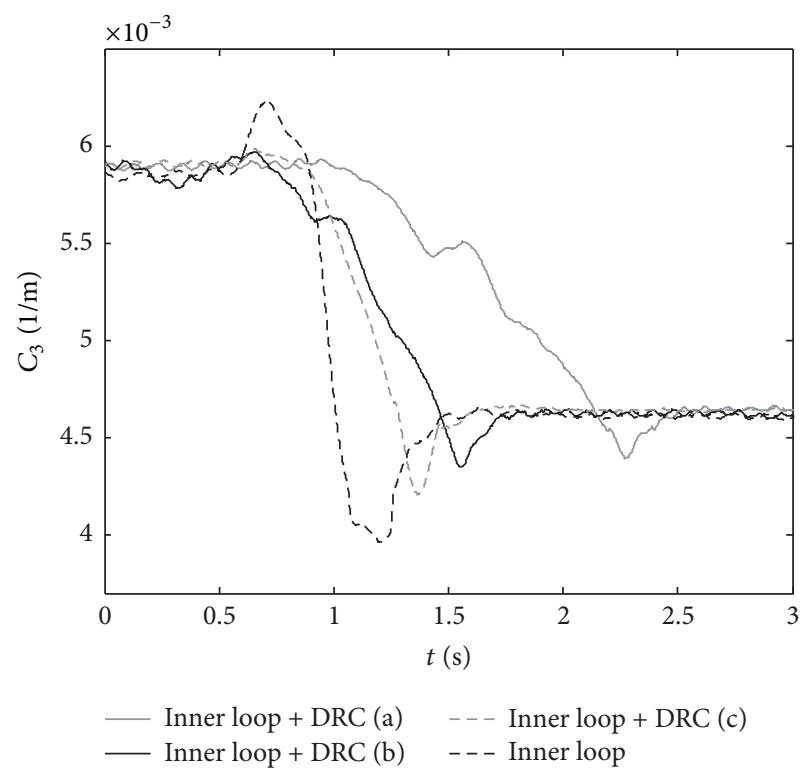

FIgURE 7: Comparison of the follower strains.

Figure 5 also shows that the presence of the high-pass filter in (16) ensures that $\tau$ does not increase before the motion starts or after reaching the final target position. Conversely, $\tau$ increases after the motion begins $(t=0.5 \mathrm{~s})$ with a variable rate, making $l$ rise at a rate that is lower than the one of time.

The measured strains are finally shown in Figure 6 (crank) and in Figure 7 (follower). It is noticeable that a very effective reduction in the link deformation is obtained through the DRC scheme: both the maximum value of the strains and their slopes are significantly reduced. In particular, the controller referred to as " $b$ " provides an excellent trade-off in reducing deformation while keeping the actual maximum delay acceptable. Only a minor overshoot on the strains of the follower (which is the link not directly actuated) and a 
small amplitude oscillation caused by the motor ripple torque (which cannot be controlled) remain uncompensated.

As for the steady values of the link strains, they are due to the static deformations induced by gravity and therefore they just depend on the mechanism configuration (and on the presence of small disturbances that justify the negligible differences). Therefore, they cannot be eliminated through the DRC, as well as through any other controller. The presence of the aforementioned high-pass filter prevents from uncontrolled growth of the time delay due to such steadystate values.

\section{Conclusions}

A control strategy for reducing elastic deformation in rest-torest motion has been proposed and experimentally validated in this paper. The technique, named Delayed Reference Control (DRC), takes advantage of the dynamic coupling between rigid-body motion and elastic motion to reduce the link deformation by suitably shaping the time history of the position reference on the basis of the sensed link strains. Being based on the measured strains, the proposed feedback scheme allows overcoming one of the main limitations of the open-loop optimal planning techniques, whose performances are affected by the presence of model uncertainty, unknown external forces, or unknown initial conditions.

Basically, the proposed DRC scheme reduces the elastic deformations by delaying or speeding up the planned reference input through an action reference parameter, which is computed in a feedback loop devoted to deformation control and closed outside a standard position control loop. The outer loop also includes the trajectory planner, which calculates the time history of the actuator position reference to be tracked by the inner position loop. Overall, this strategy allows ensuring the correct tracking of the desired spatial path while reducing the unwanted elastic deformations that usually downgrade precision and accuracy of machines. Additionally, the multiloop architecture makes the DRC implementation straightforward and suitable to be applied to manufacturing systems with proprietary controllers. Indeed, the proposed scheme can be achieved by simply adding a new loop outside proprietary position controllers.

The experimental results obtained by applying the DRC to a four-bar linkage with flexible links demonstrate the effectiveness of the approach and its stability over a wide range of gains. In particular, a significant reduction in the link unwanted elastic deformation has been achieved with a small time delay. Additionally, the results prove the correctness of some minor simplifications assumed in the formulation of the optimization problem leading to the analytical and straightforward computation of the suitable time delays. Finally, the low computational effort of the analytical solution proposed makes the DRC suitable for hard real-time implementation.

\section{Conflicts of Interest}

The authors declare that there are no conflicts of interest regarding the publication of this paper.

\section{Acknowledgments}

This research has been funded by the University of Padova through the Progetto di Ateneo 2015 CPDA157149, "efficient modeling of flexible link manipulators for real-time state estimation."

\section{References}

[1] L. Sheng, W. Li, Y. Wang, M. Fan, and X. Yang, "Dynamic model and vibration characteristics of planar 3-RRR parallel manipulator with flexible intermediate links considering exact boundary conditions," Shock and Vibration, vol. 2017, Article ID 1582547, 13 pages, 2017.

[2] P. Boscariol and A. Gasparetto, "Model-based trajectory planning for flexible-link mechanisms with bounded jerk," Robotics and Computer-Integrated Manufacturing, vol. 29, no. 4, pp. 9099, 2013.

[3] H. Karagülle, L. Malgaca, M. Dirilmiş, M. Akdağ, and S. Yavuz, "Vibration control of a two-link flexible manipulator," Journal of Vibration and Control, vol. 23, no. 12, pp. 2023-2034, 2017.

[4] T. Solatges, M. Rognant, S. Rubrecht, E. Courteille, and P. Bidaud, "Design process of high dynamics multi-link flexible robot manipulators," in Proceedings of the in Proceedings of the ASME 2017 International Design Engineering Technical Conferences Computers and Information in Engineering Conference (IDETC/CIE, Cleveland, Ohio, USA, 2017.

[5] Q. Zhang, X. Zhang, and J. Wei, "Experimental study of active vibration control of planar 3- R RR flexible parallel robots mechanism," Shock and Vibration, vol. 2016, Article ID 4780181, 17 pages, 2016.

[6] E. Lu, W. Li, X. Yang, M. Fan, and Y. Liu, "Modelling and Composite Control of Single Flexible Manipulators with Piezoelectric Actuators," Shock and Vibration, vol. 2016, Article ID 2689178, 14 pages, 2016.

[7] P. Boscariol, A. Gasparetto, and V. Zanotto, "Simultaneous position and vibration control system for flexible link mechanisms," Meccanica, vol. 46, no. 4, pp. 723-737, 2011.

[8] J. Wang, Y. Pi, Y. Hu, Z. Zhu, and L. Zeng, "Adaptive simultaneous motion and vibration control for a multi flexiblelink mechanism with uncertain general harmonic disturbance," Journal of Sound and Vibration, vol. 408, pp. 60-72, 2017.

[9] R. Caracciolo, D. Richiedei, and A. Trevisani, "Experimental validation of a model-based robust controller for multi-body mechanisms with flexible links," Multibody System Dynamics, vol. 20, no. 2, pp. 129-145, 2008.

[10] W. J. O'Connor, "Wave-based analysis and control of lumpmodeled flexible robots," IEEE Transactions on Robotics, vol. 23, no. 2, pp. 342-352, 2007.

[11] W. J. O'Connor and H. Habibi, "Wave-based control of underactuated flexible structures with strong external disturbing forces," International Journal of Control, vol. 88, no. 9, pp. 18181829, 2015.

[12] P. Gallina and A. Trevisani, "Delayed reference control of a twomass elastic system," Journal of Vibration and Control, vol. 10, no. 1, pp. 135-159, 2004.

[13] D. Richiedei and A. Trevisani, "Design of delayed reference controllers for simultaneous path tracking and active vibration damping of multi-degree-of-freedom linear systems," Journal of Vibration and Control, vol. 15, no. 3, pp. 323-346, 2009. 
[14] P. Boscariol, A. Gasparetto, R. Vidoni, and V. Zanotto, "A delayed force-reflecting haptic controller for master-slave neurosurgical robots," Advanced Robotics, vol. 29, no. 2, pp. 127-138, 2015.

[15] P. Gallina, "Delayed reference control for hotwire cutting of expandable polystyrene foam," Journal of Manufacturing Science and Engineering, vol. 128, no. 1, pp. 360-365, 2006.

[16] D. Richiedei, "Synchronous motion control of dual-cylinder electrohydraulic actuators through a non-time based scheme," Control Engineering and Applied Informatics, vol. 14, no. 4, pp. 80-89, 2012.

[17] J.-H. Park, J. Park, and S. Moon, "Real-time bilateral control for an internet-based telerobotic system," JSME International Journal Series C Mechanical Systems, Machine Elements and Manufacturing, vol. 47, no. 2, pp. 708-714, 2004.

[18] G. Boschetti, D. Richiedei, and A. Trevisani, "Delayed reference control applied to flexible link mechanisms: A scheme for effective and stable control," Journal of Dynamic Systems, Measurement, and Control, vol. 134, no. 1, Article ID 011003, 9 pages, 2012.

[19] S. Boyd and L. Vandenberghe, Convex Optimization, Cambridge University Press, Cambridge, UK, 2004.

[20] R. Caracciolo, D. Richiedei, and A. Trevisani, "Robust piecewise-linear state observers for flexible link mechanisms," Journal of Dynamic Systems, Measurement, and Control, vol. 130, no. 3, Article ID 031011, 2008. 


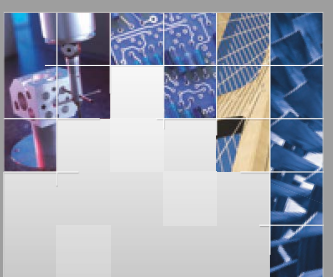

\section{Enfincering}
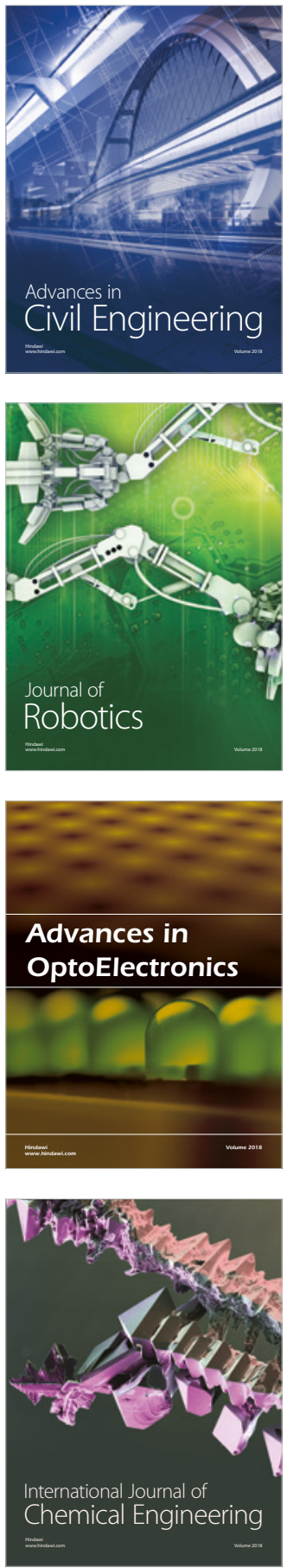

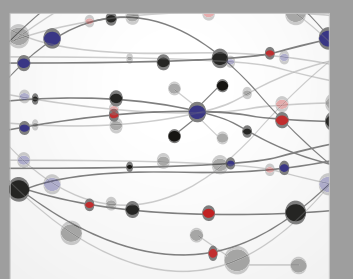

\section{Rotating \\ Machinery}

The Scientific World Journal

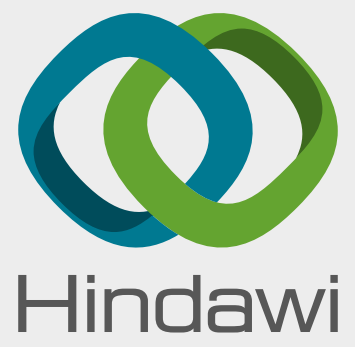

Submit your manuscripts at

www.hindawi.com
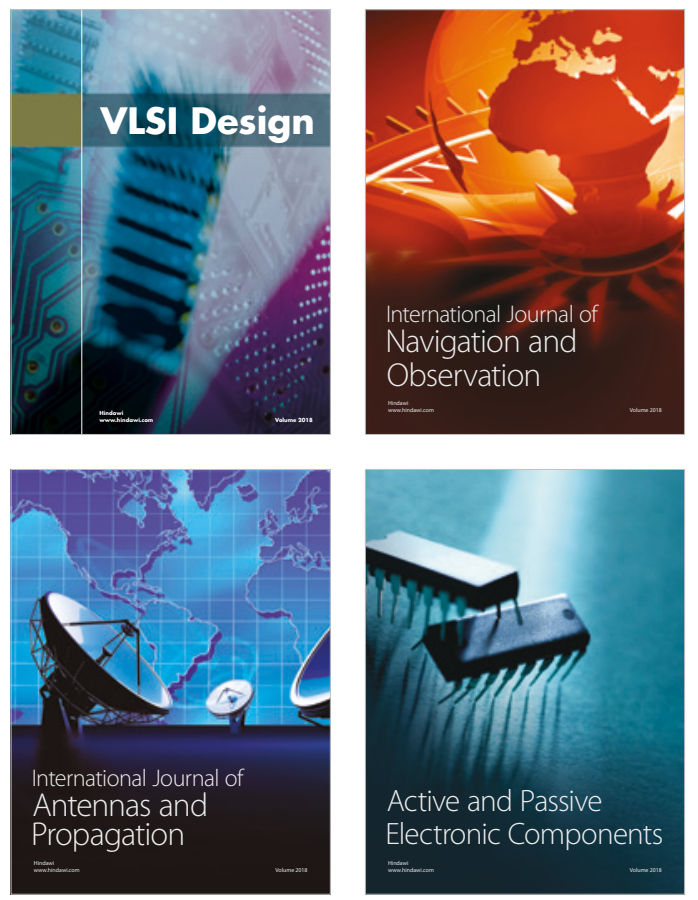
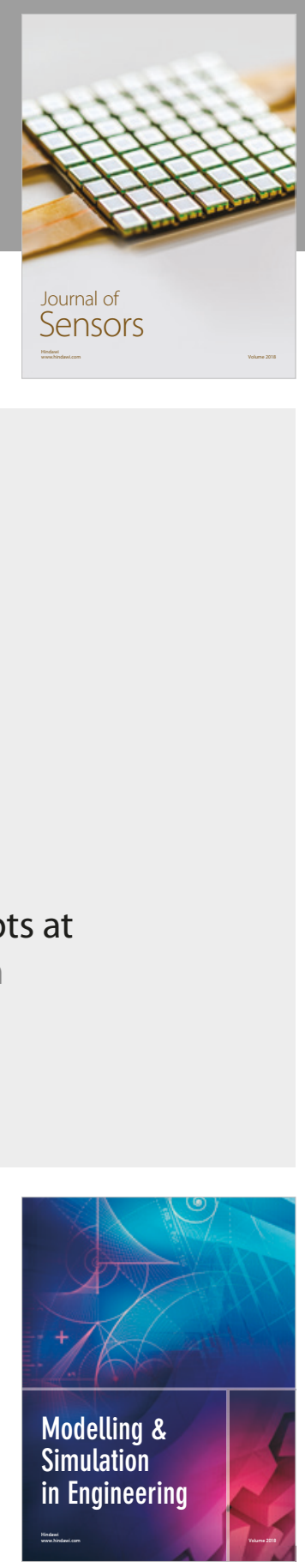

\section{Advances \\ Multimedia}
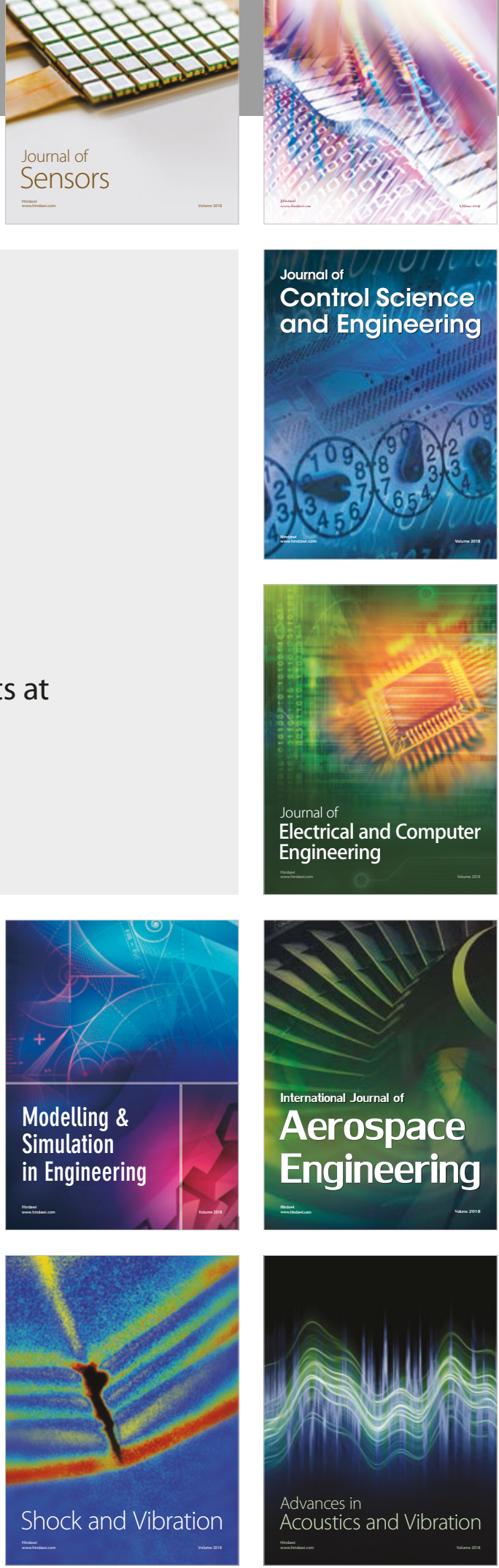